\title{
Hierarchical porous carbon spheres derived from larch sawdust via spray pyrolysis and soft-templating method for supercapacitors
}

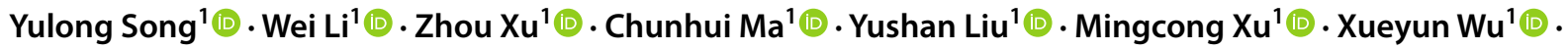 \\ Shouxin Liu ${ }^{1}$
}

(c) Springer Nature Switzerland AG 2018

\begin{abstract}
Hierarchical porous carbon spheres (PCSs) with a tunable pore structure were prepared from larch sawdust via spray pyrolysis and a soft-templating method. Decomposition of the soft template, i.e., Pluronic F127, in the carbonization step led to formation of a hierarchical porous structure with mesopores and macropores. Swelling of the core, presumably predominantly of the poly(propylene oxide) component, was induced by evaporation of water. This led to the generation of ultra-large mesopores and macropores. The surface morphology and pore structure of the carbon spheres were controlled by varying the F127 concentration. The PCSs morphologies changed from smooth to rough with increasing addition of F127, and the particle sizes became more uniform. When the content of F127 reached 0.075 (ratio of F127/ larch-based resin), the prepared PCSs (PCSs-0.075) had structural integrity, a high surface area $\left(760.3 \mathrm{~m}^{2} \mathrm{~g}^{-1}\right)$, and a large pore volume $\left(0.59 \mathrm{~cm}^{3} \mathrm{~g}^{-1}\right)$, and contained a combination of micropores, macropores, and mesopores. X-ray diffraction and Raman spectroscopy showed that addition of $\mathrm{F} 127$ had little effect on the graphitic degree of the samples. Because of its hierarchical pore structure and high surface area, the optimized sample, i.e., PCSs-0.075, gave an excellent electrochemical performance as an electrode material for supercapacitors, with a specific capacitance of $338.8 \mathrm{~F} \mathrm{~g}^{-1}$ at $0.2 \mathrm{~A} \mathrm{~g}^{-1}$ and a good rate capability $\left(165.1 \mathrm{~F} \mathrm{~g}^{-1}\right.$ at $\left.5 \mathrm{~A} \mathrm{~g}^{-1}\right)$ in $6 \mathrm{M} \mathrm{KOH}$ solution. These PCSs with mesopores and macropores, which facilitate penetration of ions into pores, are promising as efficient electrode materials for supercapacitors.
\end{abstract}

Keywords Carbon materials · Biomass · Spray pyrolysis · Pluronic F127 - Electrochemical properties

\section{Introduction}

Carbon spheres are widely used in areas such as adsorption [1-3], catalysis $[4,5]$, energy storage $[6,7]$, and electrode materials [8] because of their unique structures and properties such as a high surface area, large pore volume, good electrical conductivity, and excellent chemical stability. Recently, various methods have been used for the fabrication of carbon spheres, including hydrothermal/solvothermal [9], chemical vapor deposition [10-12], laser ablation [13], shock compression [14], and microwave-assisted methods [15]. However, there are few methods for the preparation of carbon spheres under continuous operating conditions. Spray pyrolysis is a facile method that has been widely used in the consecutive synthesis of fine particles in industry and it provides a good approach to the preparation of carbon spheres [16]. The precursor solutions are nebulized into micron-sized droplets by an ultrasound atomizer, but this is not enough to drive chemical reactions. The droplets are then carried into a furnace tube by an inert gas and the product is obtained via rapid pyrolysis. The

$\triangle$ Wei Li, liwei19820927@126.com; $₫$ Shouxin Liu, liushouxin@126.com; Yulong Song, songyulong1119@126.com; Zhou Xu, xuzhou0194@126.com; Chunhui Ma, mchmchmchmch@163.com; Yushan Liu, liuyushan_1995@126.com; Mingcong Xu, m18846145637@163.com; Xueyun Wu, 2621673415@qq.com | College of Materials Science and Engineering, Northeast Forestry University, Harbin 150040, People's Republic of China.

SN Applied Sciences (2019) 1:122 | https://doi.org/10.1007/s42452-018-0132-6 
residence time is very short, even only a few seconds. Generally, the carbon spheres produced by spray pyrolysis are largely microporous, which hinders ion diffusion and limits their electrochemical applications. It is therefore necessary to develop appropriate strategies for the preparation of porous carbon spheres (PCSs).

Much progress has been achieved in solving these problems by controlling the pore sizes of carbon spheres. Recently, many strategies have been used to fabricate PCSs, e.g., hard-templating [17] and soft-templating methods [18]. However, the hard-templating method is more time consuming and complicated than soft templating, therefore the practical applications of hard templating are limited. Soft templating has attracted much attention because it enables easy control of the morphology and pore structure of spherical particles. Soft templates generally consist mainly of emulsion droplets, micelles, vesicles, or bubbles, and template removal by carbonization is easier and safer than in the hard-templating method [19]. Commonly used carbon precursors for the soft-templated synthesis of PCSs are phenolic resin [20,21], coal [22], pitch [23], and their derivatives. Compared with conventional carbon sources, biomass has the advantages of being cheap, environmentally sustainable, and renewable. The production of carbon spheres from renewable carbon resources is therefore desirable.

Lignocellulose, which is the most abundant sustainable biomass material, consists mainly of cellulose, hemicellulose, and lignin [24]. Larch sawdust, which is a biomass feedstock, contains a large amount of lignocellulose, and is always treated as waste. Additionally, the lignin and tannin in larch contain large amounts of polyphenols, which can be a good substitute for phenolics [25]. However, the phenolic-type structures in larch are larger than phenolics and contain additional functional groups [26]. The direct synthesis of carbon spheres from larch sawdust is difficult and inefficient. The macromolecules in larch sawdust are therefore transformed into smaller molecules by liquefaction for carbon sphere preparation. We previously reported that liquefied larch could be used to fabricate carbon spheres with honeycomb-like structures through spray pyrolysis. However, the spherical structure of the carbon spheres was easily damaged, which limits their applications [27]. To obtain completely spherical structures, a larch-based resin was obtained by further resinification after liquefaction and used to fabricate carbon spheres.

In this study, PCSs with developed micropores, mesopores, and macropores were prepared from larch sawdust as the carbon precursor and F127 as a soft template via spray pyrolysis. The morphologies, particle sizes, and pore structures of the PCSs were effectively regulated by adjusting the amount of F127 in the reaction system.
Because of their unique properties, the PCSs had high specific capacitances and good rate capabilities.

\section{Materials and methods}

\subsection{Materials}

Larch sawdust (30-80 mesh) was purchased from local lumber mill, phenol (AR grade) and formaldehyde $(\mathrm{HCHO}$, $38 \%$, AR grade) were purchased from Kermel Chemical Reagent $\mathrm{Co}$ and used as received, sulfuric acid $\left(\mathrm{H}_{2} \mathrm{SO}_{4}\right.$, 98\%), phosphoric acid $\left(\mathrm{H}_{3} \mathrm{PO} 4,85 \%\right)$, methanol $\left(\mathrm{CH}_{3} \mathrm{OH}\right.$, AR grade) and sodium hydroxide ( $\mathrm{NaOH}, \mathrm{AR}$ grade) were purchased from Tianli Chemical Reagent $\mathrm{Co}$ and used as received, distilled water. Triblock copolymer Pluronic F127 [Mw = 12 600; poly(ethylene oxide) (PEO)106-poly(propylene oxide) (PPO)-70-PEO-106] was purchased from Sigma-Aldrich Corp.

\subsection{Preparation of PCSs}

A larch-based resin was prepared according to a previously reported method [28]. Typically, larch sawdust (10 g), phenol (30 mL), sulfuric acid (98 wt\%, $1 \mathrm{~mL})$, and phosphoric acid $(85 \mathrm{wt} \%, 2 \mathrm{~mL}$ ) were mixed and heated under reflux at $120^{\circ} \mathrm{C}$ for $2 \mathrm{~h}$. Methanol $(100 \mathrm{~mL})$ was added to the liquefied product. The residue was removed by filtration and sodium hydroxide solution was added until the $\mathrm{pH}$ of the mixture was neutral. The mixture was filtered again and the filtrate was concentrated by vacuum distillation at $40^{\circ} \mathrm{C}$ to achieve larch liquefaction. Finally, sodium hydroxide $(3 \mathrm{~g})$ as a catalyst and formaldehyde ( $37 \mathrm{wt} \%, 90 \mathrm{~mL}$ ) were added and the mixture was stirred at $40{ }^{\circ} \mathrm{C}$ for $20 \mathrm{~h}$ to obtain larch-based resin.

PCSs were prepared from the larch-based resin as the carbon precursor and the triblock copolymer Pluronic F127 as the soft template. As shown in Fig. 1, different amounts of $\mathrm{F} 127$ were dissolved in distilled water $(20 \mathrm{~mL})$ and mixed with the larch-based resin $(17.857 \mathrm{~g})$. Distilled water $(980 \mathrm{~mL}$ ) was added to dilute the mixture. The obtained homogeneous solution was transferred to a household humidifier (POVOS PW106, 1.7 MHz). The liquid was atomized into aerosol droplets and transported by a high-purity argon flow into a furnace tube at $900^{\circ} \mathrm{C}$. The products were collected in a flask with a condensing device at the end of the furnace. The solid particles were isolated by vacuum filtration, washed several times with distilled water and ethanol, and dried overnight at $105^{\circ} \mathrm{C}$. The obtained products were carbonized in a furnace tube in a $\mathrm{N}_{2}$ atmosphere at $500{ }^{\circ} \mathrm{C}$ for $2 \mathrm{~h}$, at a heating rate of $5^{\circ} \mathrm{C} \mathrm{min}^{-1}$, to remove the template. The samples obtained with different amounts of F127 are denoted by PCSs- $x$, 




Larch sawdust

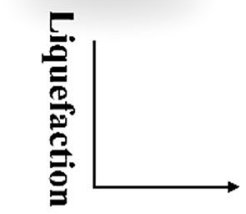

F127

Larch-based resin oligomer

\section{Larch-based resin}

F127 micelles

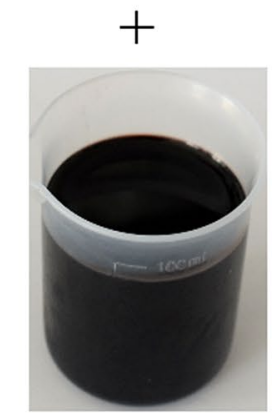

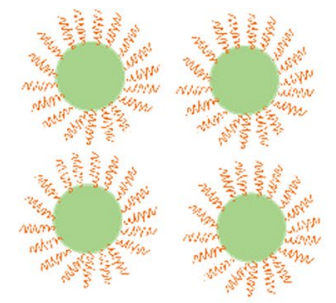

\section{$\underset{\text { Crossing-link }}{\longrightarrow}$}

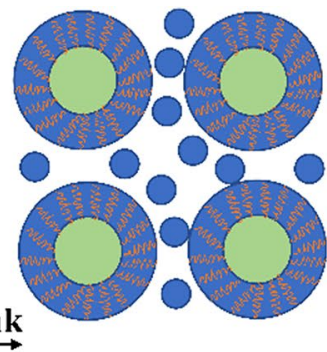

PCSs

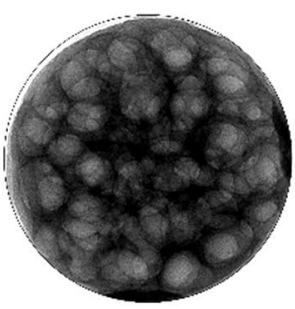

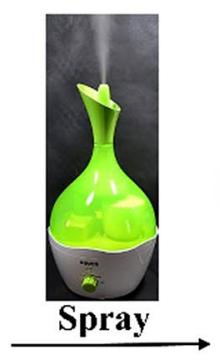

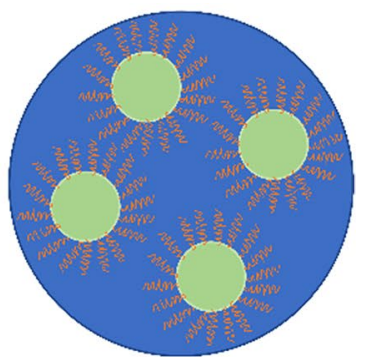

Droplets

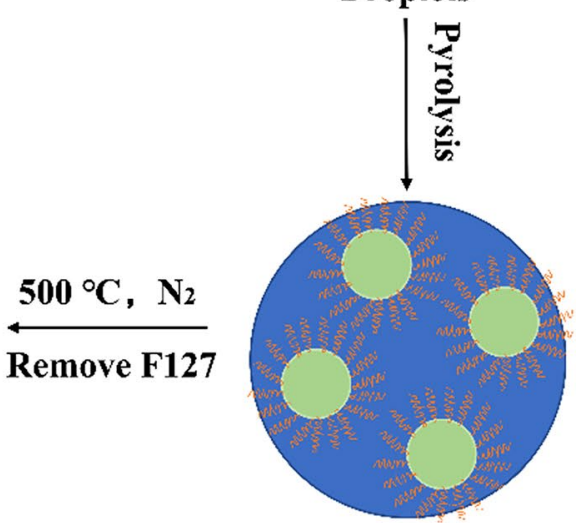

Fig. 1 Schematic diagram of formation of PCSs from larch sawdust through spray pyrolysis combined with soft templating

where $x$ is the $\mathrm{F} 127 /$ larch-based resin ratio $(x=0.05,0.075$, $0.1,0.125$, and 0.15 , respectively). A sample prepared without addition of F127 was used for comparison, and denoted by CSs.

\subsection{Characterization}

The morphologies, sizes, and structures of the products were examined by scanning electron microscopy (SEM; Quanta 200; FEl, Hillsboro, OR, USA) and transmission electron microscopy (TEM; Tecnai G2 F20 S-TWIN, USA) at an accelerating voltage of $15 \mathrm{kV}$ and $200 \mathrm{kV}$, respectively. The samples for TEM observations were dispersed in ethanol and dropped on a holey carbon film on a $\mathrm{Cu}$ grid. $\mathrm{N}_{2}$ adsorption-desorption isotherm analysis was performed at $-196{ }^{\circ} \mathrm{C}$ with a surface area and porosity analyzer (gas adsorption analyzer, Micromeritics ASAP 2020, USA). The samples were degassed at $200^{\circ} \mathrm{C}$ under vacuum for $4 \mathrm{~h}$ before the adsorption tests. The specific surface areas $\left(S_{\mathrm{BET}}\right)$ were calculated by the Brunauer-Emmett-Teller (BET) method. The total pore volume was estimated by the single-point method from the amount of $\mathrm{N}_{2}$ adsorbed at a relative pressure $\left(P / P_{0}\right)$ of 0.99 . The pore diameter distribution curve was derived from the adsorption branch by the Barret-Joyner-Halenda (BJH) method. The crystal structures of the samples were identified by powder X-ray diffractometer (XRD, Brucker D8, German) (Cu Ka radiation, $\lambda=0.15406 \mathrm{~nm}$ ), operated at a scanning rate of $5^{\circ} \mathrm{min}^{-1}$ in the $2 \theta$ range $10^{\circ}-90^{\circ}$. The disorder degrees of the samples were determined by Raman spectroscopy (Raman spectrometer, Renishaw inVia, UK) with a $633 \mathrm{~nm}$ laser as the excitation source. Fourier-transfer infrared (FT-IR) spectra were recorded with an FT-IR instrument (Perkin Elmer TV 1900 , Waltham, MA, USA) in the range $400-4000 \mathrm{~cm}^{-1}$ at a resolution of $4 \mathrm{~cm}^{-1}$. Thermogravimetric analysis (TGA) was performed with a Pyris1 TGA instrument (TG, Perkin Elmer, USA) from 30 to $800^{\circ} \mathrm{C}$ at a heating rate of $10^{\circ} \mathrm{C} \mathrm{min}^{-1}$ under a $\mathrm{N}_{2}$ flow of $20 \mathrm{~mL} \mathrm{~min}^{-1}$.

\subsection{Electrochemical characterization}

Electrochemical measurements were performed with an electrochemical work station (Solartron Analytical 1400A) in a three-electrode system with $6 \mathrm{M} \mathrm{KOH}$ as the electrolyte, a $\mathrm{Hg} / \mathrm{HgO}$ electrode as the reference electrode, and Pt wire as the counter electrode. The working electrodes were prepared by coating a viscous slurry of the active material $(16 \mathrm{mg})$, carbon black $(3 \mathrm{mg})$, and $60 \mathrm{wt} \%$ polytetrafluoroethylene $(2.0 \mu \mathrm{L})$ in a small amount of ethanol onto a Ni foam substrate. The weight of the active material loaded on each working electrode was about $5 \mathrm{mg}$. The electrodes were dried at $105^{\circ} \mathrm{C}$ for $24 \mathrm{~h}$. The electrochemical properties of the working electrode were investigated by galvanostatic charge/discharge (GCD) measurements, cyclic voltammetry (CV), and electrical impedance spectroscopy (EIS). The voltage used for the 
GCD measurements and $C V$ varied from -1 to $0 \mathrm{~V}$. The $\mathrm{CV}$ scanning rates were $5-100 \mathrm{mV} \mathrm{s}^{-1}$, and the current densities in the GCD tests were $0.2-5.0 \mathrm{~A} \mathrm{~g}^{-1}$. ElS was performed in the frequency range $10^{5}-10^{-2} \mathrm{~Hz}$ at the open-circuit voltage with an amplitude voltage of $5 \mathrm{mV}$. The specific capacitance was calculated from the GCD results according to the following equation:

$C=I \Delta t / m \Delta V$

where $C$ is the specific capacitance of the supercapacitor $\left(\mathrm{F} \mathrm{g}^{-1}\right), I$ is the charge/discharge current $(\mathrm{A}), \Delta t$ is the discharge time (s), $\Delta V$ is the GCD potential window (1.0 V), and $m$ is the mass of the active material in the electrode (g).

\section{Results and discussion}

The sizes and morphologies of the CSs and PCSs with different F127 contents were investigated by SEM. The SEM images in Fig. 2a-f show that the concentration of F127 significantly affected the particle sizes and spherical morphologies of the samples. The CSs prepared without addition of F127 were monodispersed, regular spheres with smooth surfaces, with nonuniform particles of diameter 300-900 $\mathrm{nm}$. Particle sizes are mainly determined by the droplet diameter and molar concentration of the solution. The droplet sizes produced by ultrasonication mainly depend on the atomization frequency and the properties of the precursor solution. After addition of F127, the PCS particles became uniform, which indicates that the addition of F127 affected the diameters of the carbon spheres. When the proportions of $\mathrm{F} 127$ increased to 0.075 and 0.1 , there were no noticeable changes in the uniformity and spherical integrity of the particles compared with those of PCSs- 0.05 . After the proportion of F127 added reached 0.125 , the morphology of PCSs-0.125 changed slightly, with some textural features appearing on the sphere surface. Figure $2 \mathrm{f}$ shows that with further increases in the proportion of $\mathrm{F} 127$ to 0.15 , the surfaces became uneven, with many pits and holes; this can be attributed to removal of the template.

TEM and high-resolution TEM (HRTEM) images of the CSs and PCSs are shown in Fig. 3. The CSs, i.e., the sample without F127 addition, had smooth surfaces and solid structures without visible cavities. The spheres produced when a small amount of template was added, i.e., PCSs0.05 , were completely spherical with numerous large mesopores; this shows that F127 addition changed the pore structure. With further increases in the amount of F127, flaws appeared and the surfaces became rough because of removal of F127. The pore diameters
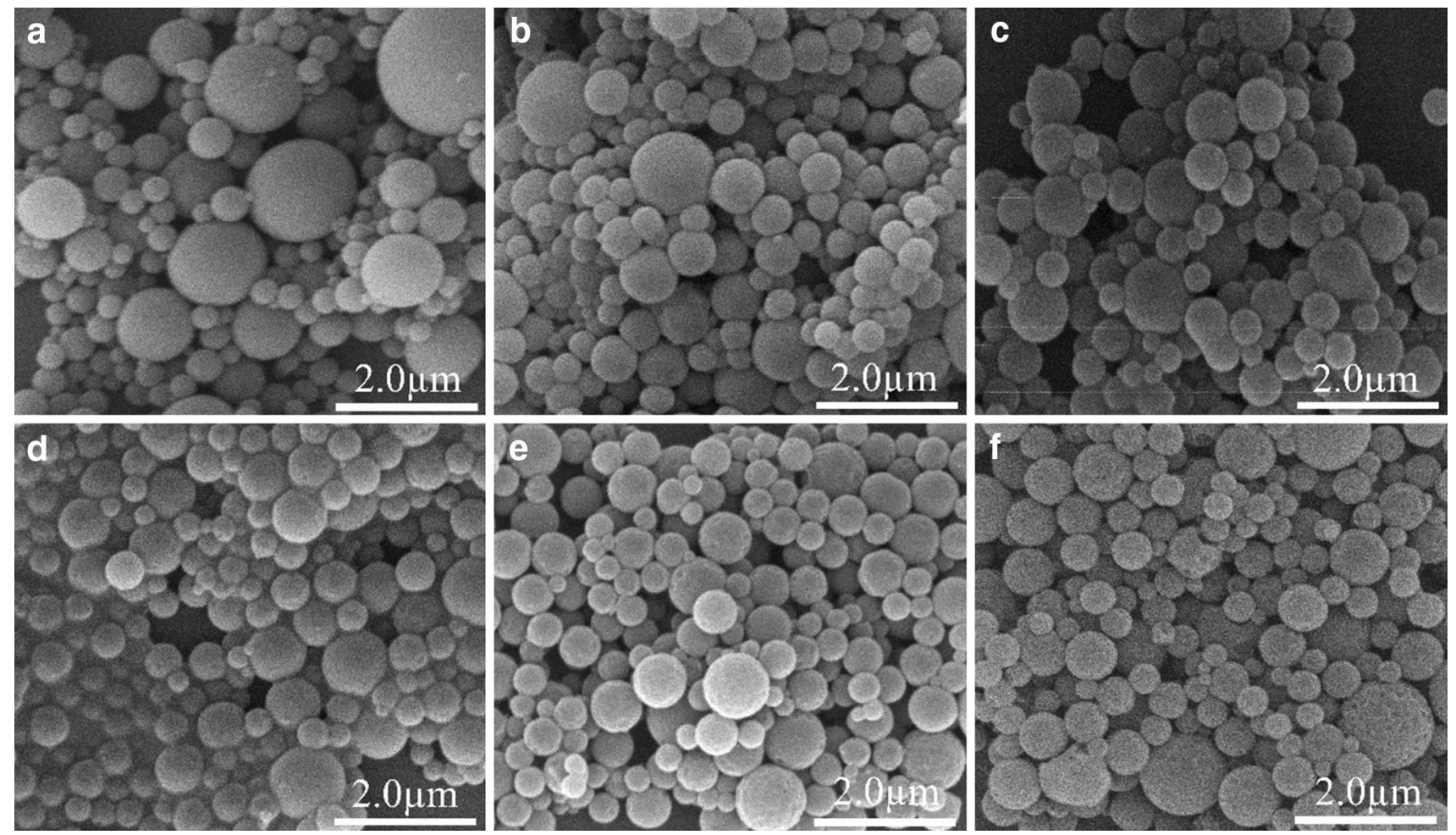

Fig. 2 SEM images of PCSs prepared with different contents of F127: a CSs; b PCSs-0.05; c PCSs-0.075; d PCSs-0.1; e PCSs-0.125; and f PCSs0.15

\section{SN Applied Sciences



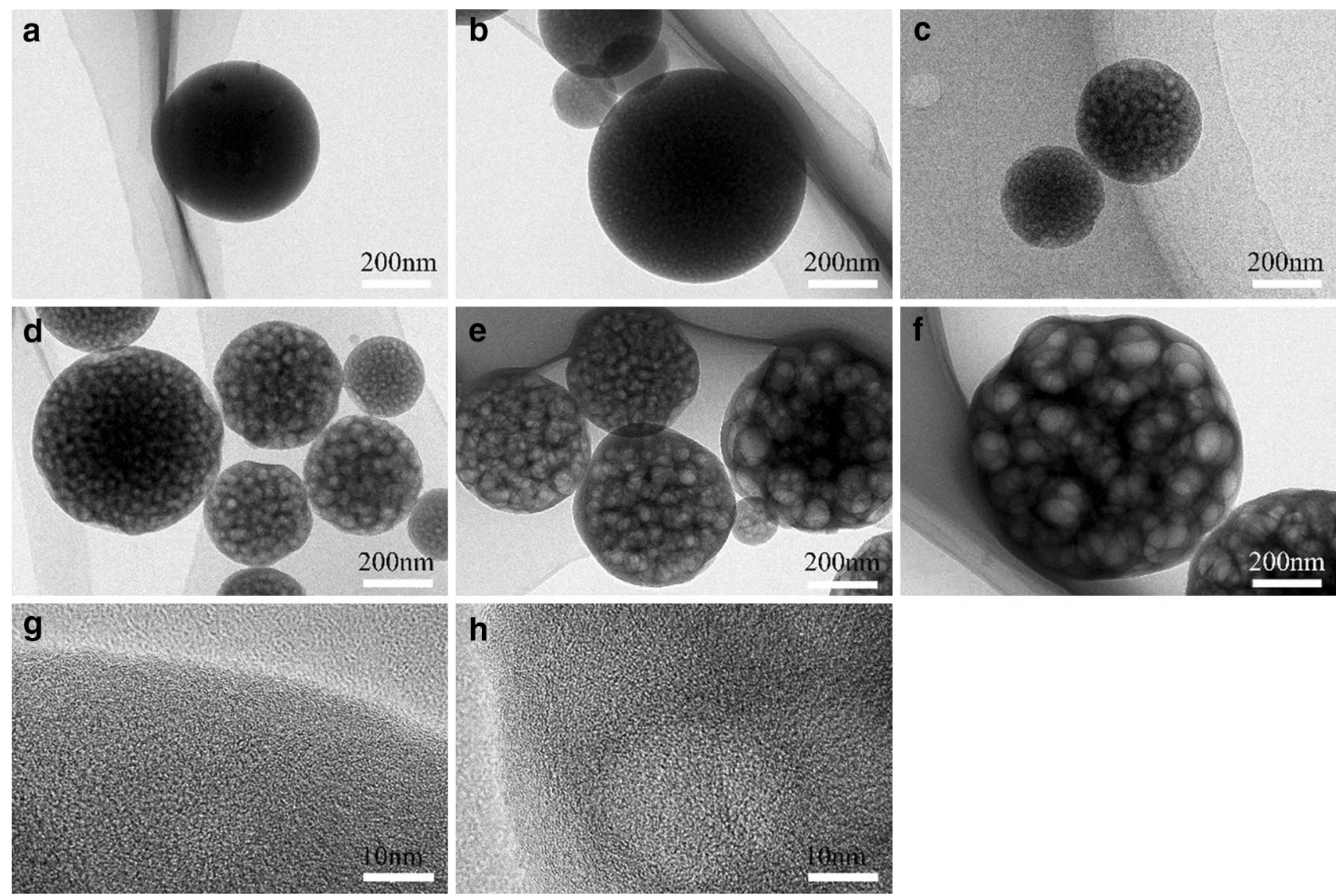

Fig. 3 TEM images of PCSs prepared with different contents of F127: a CSs; b PCSs-0.05; c PCSs-0.075; d PCSs-0.1; e PCSs-0.125; and f PCSs0.15 ; and HRTEM images of $\mathbf{g}$ CSs and $\mathbf{h}$ PCSs-0.075

increased with increasing addition of F127. The porous structures of the CSs and PCSs- 0.075 consisted of disordered, interconnected, and worm-like pores, as shown by the HRTEM images in Fig. 3g, h. Interconnected pores enhance electrolyte ion diffusion in a material. Pluronic F127 is an amphiphilic triblock copolymer and nonionic surfactant; it consists of two medium-length PEO blocks, which are hydrophilic, and a long PPO segment, which is hydrophobic [29]. In water, the PPO block is sufficiently hydrophobic relative to the PEO block to enable micelle formation [30]. Because of water evaporation during spray pyrolysis, the solution becomes more concentrated and the critical micelle concentration is reached [29, 31]. The sizes of the micelle cores increase with increasing surfactant concentration. After mixing with precursors, they usually interact with each other through van de Waals interactions and hydrogen bonds [32]. Aromatic hydrocarbons dissolved in the dense hydrophobic cores of some micelles can facilitate swelling of the micelle cores, and this increases the pore size in soft templating [33]. Disordered worm-like mesopores can be formed if condensation happens too rapidly; this is the reason for the appearance of disordered structures in the PCSs.

The $\mathrm{N}_{2}$ adsorption-desorption isotherms of the prepared CSs and PCSs are shown in Fig. 4a. The figure clearly shows that the CSs without F127 gave combined type I/IV curves, which indicates the presence of micropores and mesopores. The PCSs prepared with F127 gave type IV curves with typical H2 hysteresis loops; this shows the presence of cage-like mesopores [34]. The increases observed in the adsorption branch at $P / P_{0}=0.9-1.0$ can be ascribed to the presence of ultra-large mesopores and macropores; this is consistent with the TEM results. The mesopores in PCSs are mainly formed by removal of the soft template [35]. The area of the hysteresis loop initially increased with increasing amount of templating agent, but basically did not change when the amount of added F127 reached 0.125 . The sharp increase in the adsorption volume below a relative pressure $P / P_{0}$ of 0.1 indicates that micropores were predominant; this is consistent with the HRTEM images. The formation of micropores in the materials may be caused by evaporation of water and 

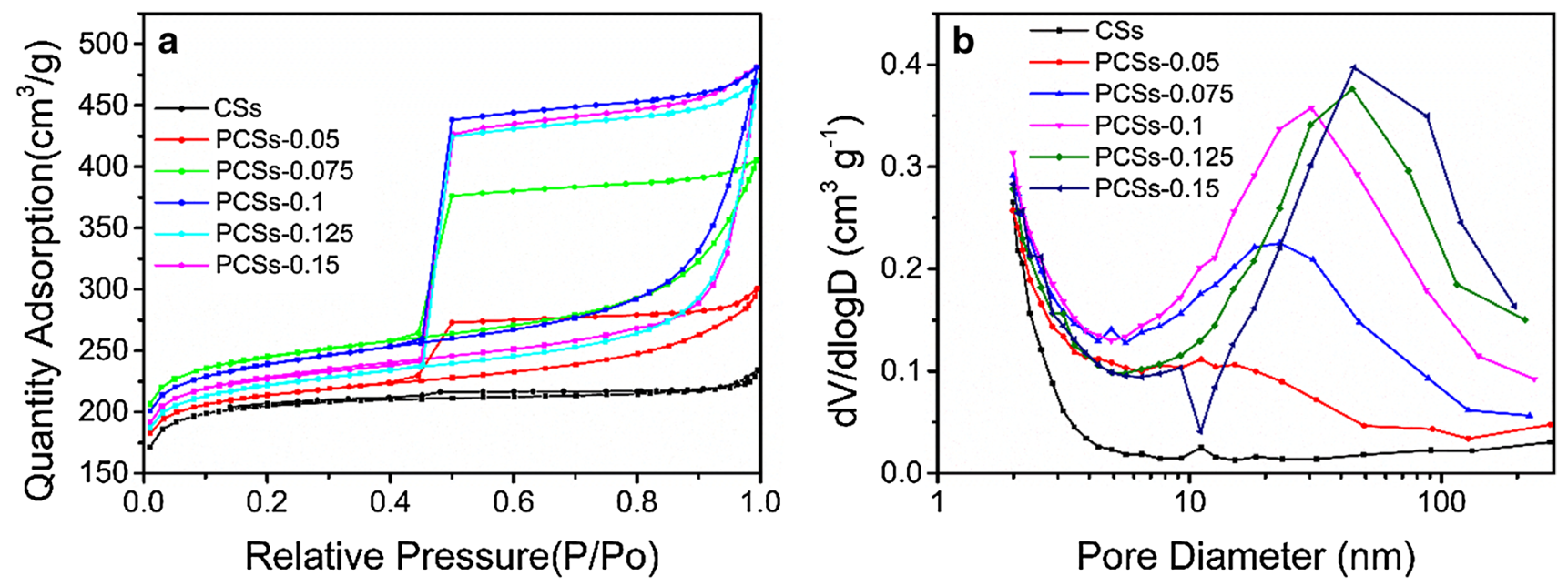

Fig. $4 \quad \mathrm{~N}_{2}$ adsorption-desorption isotherms (a) and pore size distributions (b) of CSs and PCSs prepared with different contents of F127

decomposition of oxygen-containing functional groups in the larch-based resin [36]. The pore size distribution curves calculated from the adsorption branch by the BJH method are shown in Fig. 4b. They show hierarchical pore structures with mesopores and macropores. These results are in good agreement with the $\mathrm{N}_{2}$ adsorption-desorption isotherms and TEM images.

The textural parameters of the CSs and PCSs are summarized in Table 1. The surface areas and pore volumes of the PCSs were larger than those of the CSs, i.e., those without addition of F127. The surface area and total pore volume initially increased with increasing proportion of F127. When the proportion was more than 0.075 , the surface area of the PCSs decreased slightly but the pore volume increased. This is because the increase in the concentration of the template resulted in increased amounts of mesopores and macropores.

The FT-IR spectra of the larch-based resin PCSs prepared with different amounts of F127 are shown in Fig. 5a. The spectra show that the CSs and PCSs had similar chemical structures. However, the intensity of the peak at $1650 \mathrm{~cm}^{-1}$ increased when $\mathrm{F} 127$ was added. This shows the presence of a highly conjugated carbonyl group near the hydroxyl group, and indicates that crosslinking of $\mathrm{F} 127$ with the resin enhanced the peak intensity. Pyrolysis of the CSs, PCSs-0.075, and F127 were investigated by TGA in a $\mathrm{N}_{2}$ atmosphere; the results are shown in Fig. $5 \mathrm{~b}$. In the pyrolysis of F127, weight loss occurred between 300 and $400{ }^{\circ} \mathrm{C}$, and at $400{ }^{\circ} \mathrm{C}$ the residue represented only $2.8 \mathrm{wt} \%$ of the original amount. When the temperature reached $500{ }^{\circ} \mathrm{C}$, the amount of F127 remaining was less than $1.27 \mathrm{wt} \%$. The figure shows two clear weight loss stages for the CSs and three for PCSs-0.075. Both show small weight losses below $300^{\circ} \mathrm{C}$ as a result of evaporation of organic impurities, water, and small molecules. The second stage of PCSs- 0.075 weight loss began at $300{ }^{\circ} \mathrm{C}$ and ended at $400^{\circ} \mathrm{C}$; this loss was mainly caused by thermal degradation of F127. In the last stages, the residual weights of the CSs and PCSs- 0.075 at $800{ }^{\circ} \mathrm{C}$ were $\sim 50 \%$ and $\sim 30 \%$, respectively.

Table 1 Textural properties of CSs and PCSs prepared with different contents of F127

\begin{tabular}{llllllll}
\hline Sample & $\mathrm{S}_{\text {BET }}^{\mathrm{a}}\left(\mathrm{m}^{2} \mathrm{~g}\right)$ & $\mathrm{S}_{\text {micro }}^{\mathrm{b}}\left(\mathrm{m}^{2} \mathrm{~g}\right)$ & $\mathrm{S}_{\text {meso }}^{\mathrm{c}}\left(\mathrm{m}^{2} \mathrm{~g}\right)$ & $\mathrm{V}_{\text {total }}^{\mathrm{d}}\left(\mathrm{cm}^{3} \mathrm{~g}\right)$ & $\mathrm{V}_{\text {micro }}^{\mathrm{e}}\left(\mathrm{cm}^{3} \mathrm{~g}\right)$ & $\mathrm{V}_{\text {meso }}^{\mathrm{f}}\left(\mathrm{cm}^{3} \mathrm{~g}\right)$ & $\mathrm{V}_{\text {meso }} / \mathrm{V}_{\text {total }}(\%)$ \\
\hline CSs & 626.6 & 491.9 & 134.7 & 0.34 & 0.26 & 0.08 & 23.53 \\
PCSs-0.05 & 659.0 & 491.4 & 167.6 & 0.44 & 0.26 & 0.18 & 40.91 \\
PCSs-0.075 & 760.3 & 551.1 & 209.2 & 0.59 & 0.29 & 0.30 & 50.85 \\
PCSs-0.1 & 744.3 & 519.3 & 225.0 & 0.67 & 0.27 & 0.40 & 59.70 \\
PCSs-0.125 & 689.6 & 491.3 & 198.3 & 0.60 & 0.26 & 0.34 & 56.67 \\
PCSs-0.15 & 709.4 & 509.7 & 199.7 & 0.62 & 0.27 & 0.35 & 56.45 \\
\hline
\end{tabular}

${ }^{a} \mathrm{~S}_{\mathrm{BET}}$ : specific surface area calculated by the BET method

${ }^{b},{ }^{c} S_{\text {micro, }} \mathrm{S}_{\text {meso }}$ : specific surface area of microstructure and mesostructure

${ }^{\mathrm{d}} \mathrm{V}_{\text {total }}$ : total pore volume at $P / P_{0}=0.99$

e,f $\mathrm{V}_{\text {micro }}, \mathrm{V}_{\text {meso }}$ : micropore volume and mesopore volume 

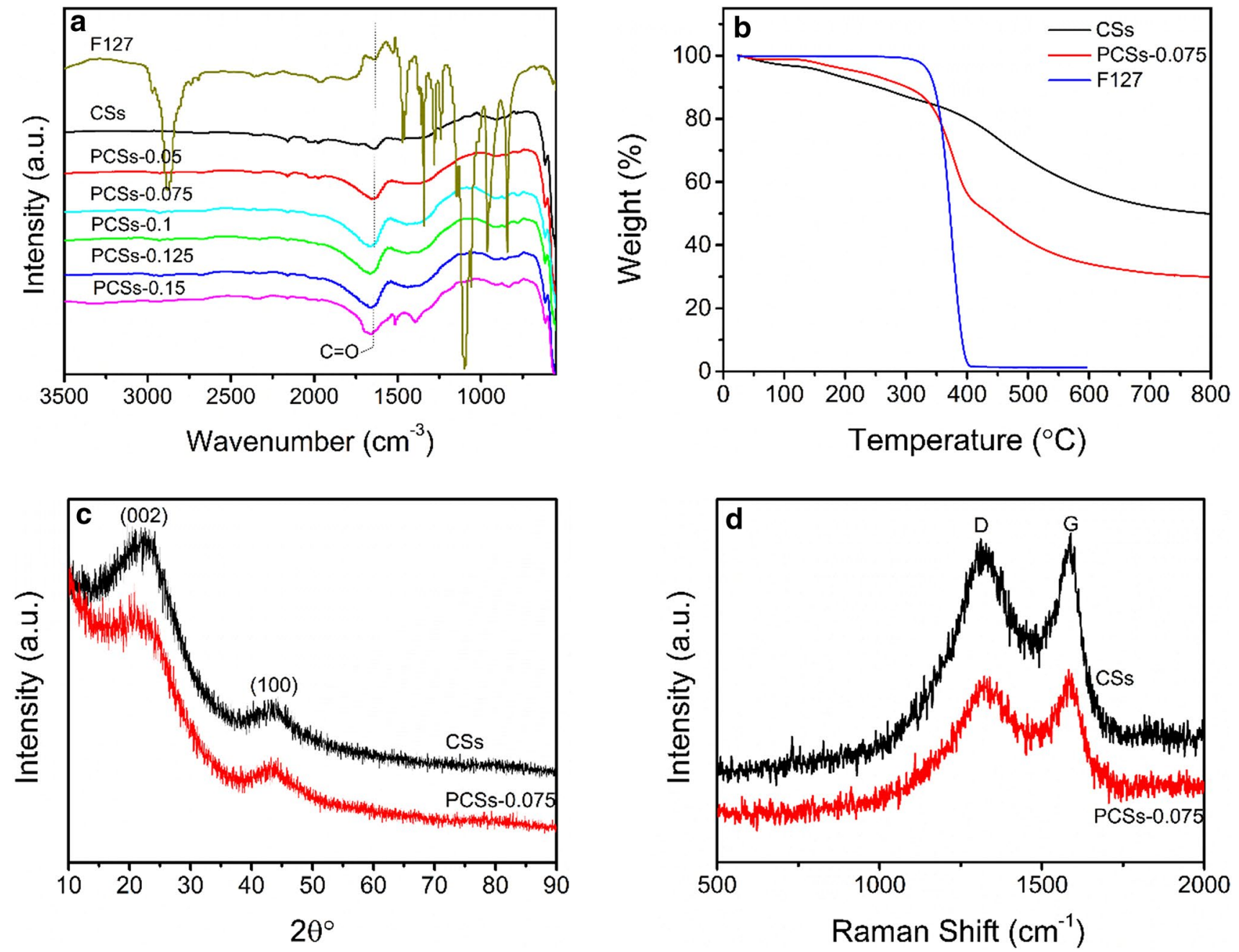

Fig. 5 FT-IR spectra of CSs and PCSs-0.075 prepared with different contents of F127 (a); and TGA curves under $\mathrm{N}_{2}$ (b); Raman spectra (c); and wide-angle XRD patterns (d) of CSs and PCSs-0.075

The lower initial weight loss for PCSs- 0.075 can be attributed to crosslinking between the larch-based resin and F127, which is consistent with the formation of a carbon skeleton with high thermal stability.

The wide-angle XRD patterns of the CSs and PCSs0.075 are shown in Fig. $5 \mathrm{c}$. The patterns for the CSs and PCSs- 0.075 contain two broad diffraction peaks, at $2 \theta$ values around $22.3^{\circ}$ and $43.6^{\circ}$, which are indexed to the (002) plane of graphitic carbon and the (100) diffraction of amorphous graphitic carbon. The peaks are broad and weak, which indicates a low degree of graphitization; this agrees well with the HRTEM results. Raman spectroscopy was used to determine the amorphous degree of the CSs and PCSs from the ratio of the D-band and G-band intensities $\left(I_{\mathrm{D}} / I_{\mathrm{G}}\right)$. Figure $5 \mathrm{~d}$ clearly shows that the spectra of the CSs and PCSs- 0.075 contain two clear peaks, at 1328 and $1590 \mathrm{~cm}^{-1}$, corresponding to the $D$ and $G$ bands. The $I_{D} / I_{G}$ values for the CSs and PCSs-0.075 are 0.99 and 0.98 , respectively. This indicates that addition of F127 had little effect on the crystallization degree of the carbon spheres.

Because of the high surface areas and hierarchical pore size distribution, $\mathrm{PCSs}-0.075$ gave excellent electrochemical performances. The samples were used as electrode materials and their performances as supercapacitors were investigated by CV and GCD measurements in a threeelectrode system with $6 \mathrm{M} \mathrm{KOH}$ as the electrolyte. The CV curves for PCSs- 0.075 at various scanning rates between 5 and $100 \mathrm{mV} \mathrm{s}^{-1}$ over the range -1.0 to $0 \mathrm{~V}$ are shown in Fig. 6a. The CV curves for the PCSs-0.075 electrode retained a rectangular-like shape with increasing scanning rate, even at $100 \mathrm{mV} \mathrm{s}^{-1}$. This corresponds to double-layer capacitance behavior. GCD measurements were also used to test the capacitor performances. The symmetry of the charge and discharge processes shows that the electrode had high electrochemical reversibility. The GCD curves of the PCSs- 0.075 electrode at current densities from 0.2 to 

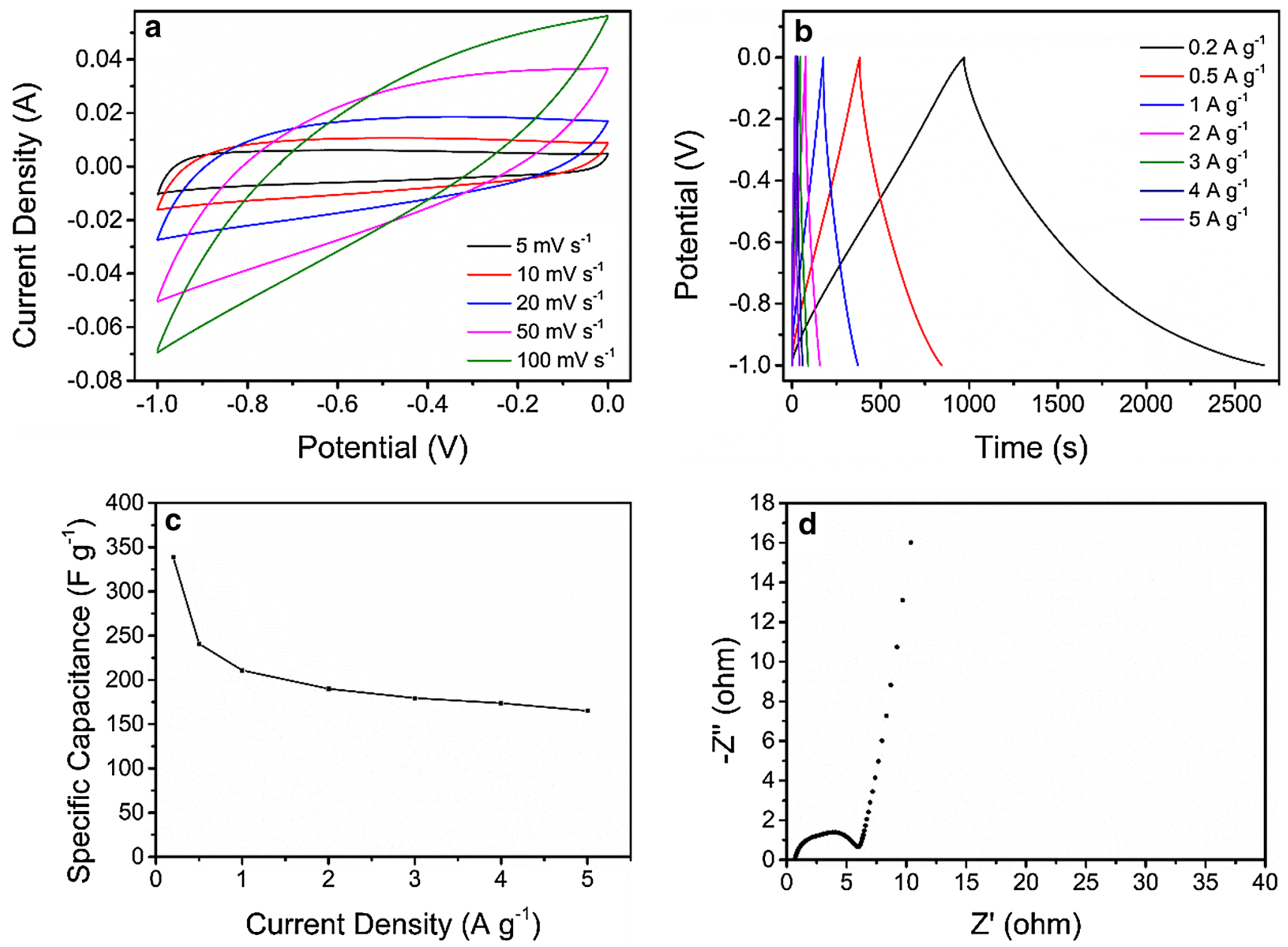

Fig. 6 a Cyclic voltammograms for PCSs-0.075 at 5-100 $\mathrm{mV} \mathrm{s}^{-1}$; b GCD curves for PCSs-0.075 at 0.2-5 A g ${ }^{-1}$; c specific capacitances of PCSs0.075 electrode at different current densities; and $\mathbf{d}$ Nyquist plots for PCSs- 0.075 electrode

Table 2 Comparison of the specific capacitance of the other carbon materials

\begin{tabular}{|c|c|c|c|c|c|}
\hline Material & Method & $\mathrm{S}_{\mathrm{BET}}\left(\mathrm{m}^{2} \mathrm{~g}^{-1}\right)$ & Electrolyte & Specific capacitance $\left(\mathrm{F} \mathrm{g}^{-1}\right)$ & Ref \\
\hline $\begin{array}{l}\text { Micro- and mesoporous activated } \\
\text { carbon }\end{array}$ & Template and $\mathrm{CO}_{2}$ activation & 1573 & $1 \mathrm{M} \mathrm{H}_{2} \mathrm{SO}_{4}$ & $106\left(5 \mathrm{mV} \mathrm{s}^{-1}\right)$ & [37] \\
\hline Porous carbon spheres & Spray drying & 1372.87 & $3 \mathrm{M} \mathrm{KOH}$ & $340\left(0.5 \mathrm{~A} \mathrm{~g}^{-1}\right)$ & [38] \\
\hline $\begin{array}{l}\text { Nitrogen-containing Hierarchical } \\
\text { porous carbon spheres }\end{array}$ & Carbonization & 1939 & $7 \mathrm{M} \mathrm{KOH}$ & $276\left(0.1 \mathrm{~A} \mathrm{~g}^{-1}\right)$ & [39] \\
\hline Hierarchical porous carbon spheres & Hard template method & 2489 & $6 \mathrm{M} \mathrm{KOH}$ & $167\left(0.2 \mathrm{~A} \mathrm{~g}^{-1}\right)$ & [40] \\
\hline Activated carbons & Hydrothermal and activation & 2967 & $1 \mathrm{MTE}-\mathrm{ABF}_{4} / \mathrm{AN}$ & $236\left(0.1 \mathrm{~A} \mathrm{~g}^{-1}\right)$ & [41] \\
\hline $\begin{array}{l}\text { Nitrogen-doped hollow Mesoporous } \\
\text { carbon spheres }\end{array}$ & Template & 1158 & $6 \mathrm{M} \mathrm{KOH}$ & $159\left(1 \mathrm{Ag}^{-1}\right)$ & [7] \\
\hline $\mathrm{N}$-doped carbon spheres & Template & 1126.11 & $6 \mathrm{M} \mathrm{KOH}$ & $323.2\left(0.2 \mathrm{~A} \mathrm{~g}^{-1}\right)$ & [42] \\
\hline $\begin{array}{l}\text { Nitrogen- and oxygen-containing } \\
\text { micro-Mesoporous carbon micro- } \\
\text { spheres }\end{array}$ & Hydrothermal and template & 3203 & $6 \mathrm{M} \mathrm{KOH}$ & $309\left(0.1 \mathrm{~A} \mathrm{~g}^{-1}\right)$ & [43] \\
\hline $\begin{array}{l}\text { Nitrogen-doped hollow carbon } \\
\text { spheres }\end{array}$ & Carbonization & 124 & $6 \mathrm{M} \mathrm{KOH}$ & $122\left(0.5 \mathrm{~A} \mathrm{~g}^{-1}\right)$ & [44] \\
\hline Porous carbon spheres & Aerosol and activation & 1610 & $6 \mathrm{M} \mathrm{KOH}$ & $219\left(0.5 \mathrm{~A} \mathrm{~g}^{-1}\right)$ & {$[45]$} \\
\hline Hierarchical porous carbon spheres & Spray pyrolysis and template & 760.3 & $6 \mathrm{M} \mathrm{KOH}$ & $338.8\left(0.2 \mathrm{~A} \mathrm{~g}^{-1}\right)$ & This work \\
\hline
\end{tabular}


$5 \mathrm{~A} \mathrm{~g}^{-1}$ are shown in Fig. 6b. The specific capacitance of PCSs- 0.075 at a current density of $0.2 \mathrm{~A} \mathrm{~g}^{-1}$, determined from the discharge process, was $338.8 \mathrm{~F} \mathrm{~g}^{-1}$. This high specific capacitance can be attributed to the high surface area and hierarchical pore structure of PCSs-0.075. The high surface area mainly attributed to micropores supplies abundant active sites, and the mesopores and macropores facilitate ion transport and shorten the diffusion distance for electrolyte ions. Figure $6 \mathrm{c}$ shows that PCSs- 0.075 at $0.5,1,2,3,4$, and $5 \mathrm{~A} \mathrm{~g}^{-1}$ showed high capacitance retention, i.e., $71.1 \%, 62.2 \%, 56.1 \%, 53.0 \%, 51.3 \%$, and $48.7 \%$, respectively, of the value at $0.2 \mathrm{~A} \mathrm{~g}^{-1}$. This indicates good capacitance retention by PCSs-0.075. Comparison of the specific capacitance of the other carbon materials is listed in Table 2, the specific capacitance of PCSs was in the high level under the current density of $0.2 \mathrm{~A} \mathrm{~g}^{-1}$. EIS was performed to further explore the supercapacitive behavior of the PCSs-0.075 electrode. The Nyquist plots for the carbon electrode (Fig. 6d) show an approximately vertical line in the low-frequency region because of Warburg impedance. This indicates excellent electrolyte ion diffusion to the electrode interface because of the highly porous nature of the electrode material.

\section{Conclusions}

In this work, a facile strategy that involved a combination of spray pyrolysis and soft templating was used to fabricate hierarchical PCSs. The carbon source was larch sawdust and the soft template was Pluronic F127. The morphologies and pore structure of the PCSs were regulated by simply adjusting the $\mathrm{F} 127 /$ larch-based resin ratio. After addition of F127, the pores became larger and the particle sizes became uniform. With further increases in the amount of F127, many pits and holes appeared on the surfaces of the PCSs. The obtained PCSs- 0.075 had high surface areas, up to $760.3 \mathrm{~m}^{2} \mathrm{~g}^{-1}$, and tunable pore sizes, with micropores, mesopores, and macropores present. The large surface area which was mainly derived from micropores and macro/mesoporous structures, increased the number of active sites and shortened the ion diffusion distance; these are favorable for achieving excellent electrochemical performances. Electrochemical measurements showed that the PCSs- 0.075 sample had a high specific capacitance of $338.8 \mathrm{~F} \mathrm{~g}^{-1}$ at $0.2 \mathrm{~A} \mathrm{~g}^{-1}$, and a good rate capability. These results show that PCSs with micropores, mesopores, and macropores can be obtained from renewable resources, and are promising electrode materials for supercapacitors.

Acknowledgements The present work was financially supported by the National Key R\&D Program of China (2017YFD0601006), the
National Natural Science Foundation of China (31570567, 31500467) and the Fundamental Research Funds for the Central Universities (2572017ET02).

\section{Compliance with ethical standards}

Conflict of interest The authors declare there is no conflict of interest regarding the publication of this paper.

\section{References}

1. Liu L, Xie ZH, Deng QF, Hou XX, Yuan ZY (2017) One-pot carbonization enrichment of nitrogen in microporous carbon spheres for efficient $\mathrm{CO}_{2}$ capture. J Mater Chem A 5(1):418-425

2. Li YQ, Yu YF, Liu L, Zhang HL, Wang GX, Chen AB (2017) Synthesis of $\mathrm{N}$-doped carbon spheres using extended stöber method for $\mathrm{SO}_{2}$ adsorption. NANO 12(01):1750004

3. Choma J, Jedynak K, Fahrenholz W, Ludwinowicz J, Jaroniec M (2014) Microporosity development in phenolic resin-based mesoporous carbons for enhancing $\mathrm{CO}_{2}$ adsorption at ambient conditions. Appl Surf Sci 289:592-600

4. Shi QR, Zhu CZ, Engelhard MH, Du D, Lin YH (2017) Highly uniform distribution of pt nanoparticles on $n$-doped hollow carbon spheres with enhanced durability for oxygen reduction reaction. RSC Adv 7(11):6303-6308

5. Yang T, Liu J, Zhou R, Chen Z, Xu H, Qiao SZ, Monteiro MJ (2014) $\mathrm{N}$-doped mesoporous carbon spheres as the oxygen reduction reaction catalysts. J Mater Chem A 2(42):18139-18146

6. Zhao HY, Zhang F, Zhang SM, He SN, Shen F, Han XG, Yin YD, Gao CB (2018) Scalable synthesis of sub-100 nm hollow carbon nanospheres for energy storage applications. Nano Res 11(4):1822-1833

7. Chen AB, Li YQ, Liu L, Yu YF, Xia KC, Wang YY, Li SH (2017) Controllable synthesis of nitrogen-doped hollow mesoporous carbon spheres using ionic liquids as template for supercapacitors. Appl Surf Sci 393:151-158

8. Tang HM, Yan D, Lu T, Pan LK (2017) Sulfur-doped carbon spheres with hierarchical micro/mesopores as anode materials for sodium-ion batteries. Electrochim Acta 241:63-72

9. Zhang ZL, Qin ML, Jia BR, Zhang HZ, Wu HY, Qu XH (2017) Facile synthesis of novel bowl-like hollow carbon spheres by the combination of hydrothermal carbonization and soft templating. Chem Commun 53(20):2922-2925

10. Zhang L, Dou YH, Guo HP, Zhang BW, Liu XX, Wan M, Li WJ, Hu XL, Dou SX, Huang YH, Liu HK (2017) A facile way to fabricate double-shell pomegranate-like porous carbon microspheres for high-performance Li-ion batteries. J Mater Chem A 5(24):12073-12079

11. Tetana ZN, Mhlanga SD, Coville NJ (2017) Chemical vapour deposition syntheses and characterization of boron-doped hollow carbon spheres. Diam Relat Mater 74:70-80

12. Xia YD, Mokaya R (2004) Ordered mesoporous carbon hollow spheres nanocast using mesoporous silica via chemical vapor deposition. Adv Mater 16(11):886-891

13. Yang SK, Zeng HB, Zhao HP, Zhang HW, Cai WP (2011) Luminescent hollow carbon shells and fullerene-like carbon spheres produced by laser ablation with toluene. J Mater Chem 21(12):4432

14. Niwase K, Homae T, Nakamura KG, Kondo K (2002) Generation of giant carbon hollow spheres from c60 fullerene by shockcompression. Chem Phys Lett 362(1):47-50

15. Zou SM, Xu XY, Zhu YQ, Cao CB (2017) Microwave-assisted preparation of hollow porous carbon spheres and as anode 
of lithium-ion batteries. Microporous Mesoporous Mater 251:114-121

16. Bang JH, Suslick KS (2010) Applications of ultrasound to the synthesis of nanostructured materials. Adv Mater 22(10):1039-1059

17. Wang GX, Wang RC, Liu L, Zhang HL, Du J, Zhang YT, Liu M, Liang $\mathrm{KH}$, Chen A (2017) Synthesis of hollow mesoporous carbon spheres via friedel-crafts reaction strategy for supercapacitor. Mater Lett 197:71-74

18. Jiao J, Xiang YQ, Cao J, Xia Y (2016) Worm-hole structured mesoporous carbon monoliths synthesized with amphiphilic triblock copolymer. J Porous Mater 23(6):1431-1438

19. Li SJ, Pasc A, Fierro V, Celzard A (2016) Hollow carbon spheres, synthesis and applications-a review. J Mater Chem A 4(33):12686-12713

20. Arif AF, Balgis R, Ogi T, Mori T, Okuyama K (2015) Experimental and theoretical approach to evaluation of nanostructured carbon particles derived from phenolic resin via spray pyrolysis. Chem Eng J 271:79-86

21. Jokić B, Drmanić S, Radetic T, Krstić J, Petrović R, Orlović A, Janaćković D (2010) Synthesis of submicron carbon spheres by the ultrasonic spray pyrolysis method. Mater Lett 64(20):2173-2176

22. Guo MX, Guo JX, Tong FL, Jia DZ, Jia W, Wu JB, Wang LX, Sun ZP (2017) Hierarchical porous carbon spheres constructed from coal as electrode materials for high performance supercapacitors. RSC Adv 7(72):45363-45368

23. Ma QT, Wang LX, Xia W, Jia DZ, Zhao ZB (2016) Nitrogen-doped hollow amorphous carbon spheres@graphitic shells derived from pitch: new structure leads to robust lithium storage. Chem Eur J 22(7):2339-2344

24. Delidovich I, Hausoul PJ, Deng L, Pfutzenreuter R, Rose M, Palkovits $R$ (2016) Alternative monomers based on lignocellulose and their use for polymer production. Chem Rev 116(3):1540-1599

25. Qin HF, Jian RH, Bai J, Tang JR, Zhou Y, Zhu BL, Zhao DJ, Ni ZJ, Wang LB, Liu WQ, Zhou QF, Li X (2018) Influence of molecular weight on structure and catalytic characteristics of ordered mesoporous carbon derived from lignin. ACS Omega 3(1):1350-1356

26. Libbrecht W, Verberckmoes A, Thybaut JW, Van Der Voort P, De Clercq J (2017) Soft templated mesoporous carbons: tuning the porosity for the adsorption of large organic pollutants. Carbon 116:528-546

27. Zhao X, Li W, Zhang SS, Liu LH, Liu SX (2015) Facile fabrication of hollow and honeycomb-like carbon spheres from liquefied larch sawdust via ultrasonic spray pyrolysis. Mater Lett 157:135-138

28. Zhao X, Li W, Liu SX (2014) Ordered mesoporous carbon membrane prepared from liquefied larch by a soft method. Mater Lett 126:174-177

29. Mai YY, Eisenberg A (2012) Self-assembly of block copolymers. Chem Soc Rev 41(18):5969-5985

30. Wang SX, Chen S, Wei Q, Zhang X, Wong SY, Sun S, Li X (2014) Bioinspired synthesis of hierarchical porous graphitic carbon spheres with outstanding high-rate performance in lithium-ion batteries. Chem Mater 27(1):336-342
31. Skrabalak SE, Suslick KS (2006) Porous carbon powders prepared by ultrasonic spray pyrolysis. J Am Chem Soc 128(39):12642-12643

32. Xiao PW, Guo D, Zhao L, Han BH (2016) Soft templating synthesis of nitrogen-doped porous hydrothermal carbons and their applications in carbon dioxide and hydrogen adsorption. Microporous Mesoporous Mater 220:129-135

33. Petkovich ND, Stein A (2013) Controlling macro- and mesostructures with hierarchical porosity through combined hard and soft templating. Chem Soc Rev 42(9):3721-3739

34. Kruk M, Jaroniec M (2001) Gas adsorption characterization of ordered organic-inorganic nanocomposite materials. Chem Mater 13(10):3169-3183

35. Qiang Z, Liu XY, Zou F, Cavicchi KA, Zhu Y, Vogt BD (2017) Bimodal porous carbon-silica nanocomposites for Li-ion batteries. J Phys Chem C 121(31):16702-16709

36. Li W, Huang ZH, Wu Y, Zhao X, Liu SX (2015) Honeycomb carbon foams with tunable pore structures prepared from liquefied larch sawdust by self-foaming. Ind Crop Prod 64:215-223

37. Kumagai S, Sato M, Tashima D (2013) Electrical double-layer capacitance of micro- and mesoporous activated carbon prepared from rice husk and beet sugar. Electrochim Acta 114:617-626

38. Chen YM, Zhang GX, Zhang JY, Guo HB, Feng X, Chen YG (2018) Synthesis of porous carbon spheres derived from lignin through a facile method for high performance supercapacitors. J Mater Sci Technol 34:2189-2196

39. Pang J, Zhang WF, Zhang $\mathrm{H}$, Zhang JL, Zhang HM, Cao GP, Han MF, Yang YS (2018) Sustainable nitrogen-containing hierarchical porous carbon spheres derived from sodium lignosulfonate for high-performance supercapacitors. Carbon 132:280-293

40. Zhou M, Lu Y, Chen HM, Ju XX, Xiang F (2018) Excellent durable supercapacitor performance of hierarchical porous carbon spheres with macro hollow cores. J Energy Storage 19:35-40

41. LuW, Marta S, Antonio BF, Robert M, Gleb Y (2011) Hydrothermal carbonization of abundant renewable natural organic chemicals for high-performance supercapacitor electrodes. Adv Energy Mater 1:356-361

42. Sun ZS, Shen SD, Ma LM, Mao DS, Lu GZ (2016) Controlled synthesis of $\mathrm{N}$-doped carbon spheres with different morphology for supercapacitor. RSC Adv 6:104642-104647

43. Liu SP, Chen XJ, Li XL, Huo PF, Wang YQ, Bai L, Zhang W, Niu MC, Li ZG (2016) Nitrogen- and oxygen-containing micro-mesoporous carbon microspheres derived from $\mathrm{m}$-aminophenol formaldehyde resin for supercapacitor with high rate performance. RSC Adv 6:89744-89756

44. Liu L, Xu SD, Yu Q, Wang FY, Zhu HL, Zhang RL, Liu X (2016) Nitrogen-doped hollow carbon spheres with wrinkled surface: one-pot carbonization synthesis and supercapacitor properties. Chem Comm 52:11693-11696

45. Tang ZH, Jiang S, Shen SL, Yang JH (2018) The preparation of porous carbon spheres with hierarchical pore structure and the application for high-performance supercapacitors. J Mater Sci 53(19):13987-14000 